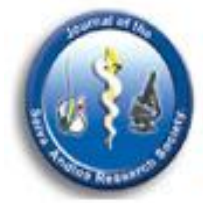

\title{
Selección de cepas de Trichoderma spp. generadoras de metabolitos secundarios de interés para su uso como promotor de crecimiento en plantas cultivadas
}

\section{Selecting strains of Trichoderma spp. generating secondary metabolites of interest for use as a} growth promoter in plants grown

\author{
Ortuño Noel ${ }^{1 *}$, Miranda Claudia ${ }^{2}$, Claros Mayra $^{1}$
}

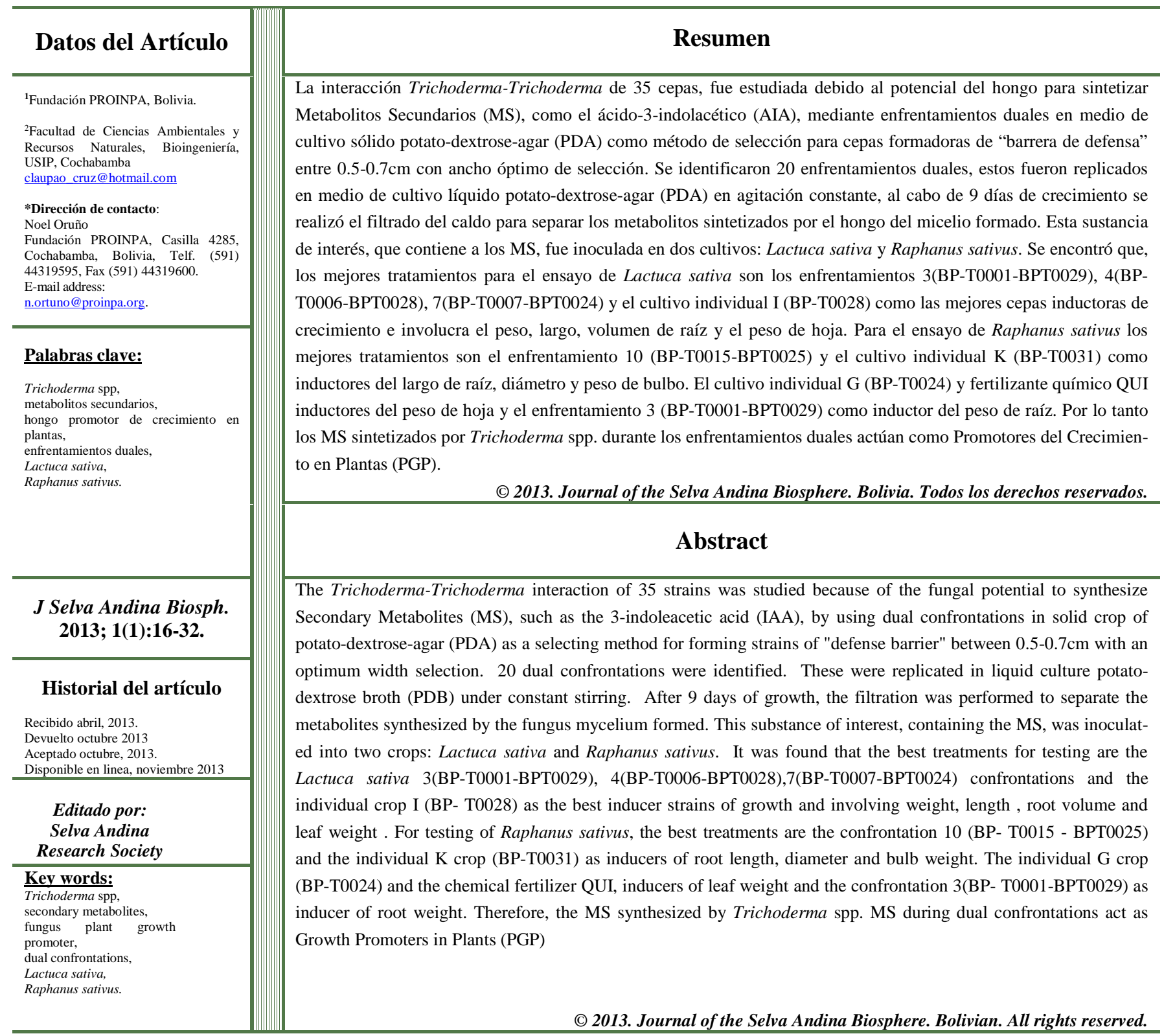




\section{Introducción}

Trichoderma es un género de hongos que tiene bastante importancia para la vida humana y la funcionalidad de un ecosistema, descomponedor de la materia orgánica, esencial en la recirculación de nutrientes en el medio ambiente. Algunos miembros de este género tienen asociaciones simbióticas con plantas, leguminosas, gramíneas, compuestas, solanaceas y otras, mientras otras son utilizados como biocontroladores contra organismos patógenos como Fusarium y Rizoctonia, además la producción de enzimas industriales como los pigmentos de antraquinona y otros (Druzhinina \& Kubicek 2005) y metabolitos secundarios (MS) como antibióticos y promotores del crecimiento de plantas (PGP: Plant Growth Promoting), ideales para la agricultura (Harman et al. 2004, Vinale et al. 2006, Vinale et al. 2008, Vinale et al. 2009, Druzhinina et al. 2011). Trichoderma spp. uno de los hongos que está presente en todo tipo de suelos agrícolas y ecosistemas, su versatilidad, adaptación y fácil manipulación (Fernández-Larrea 2001) ha permitido su uso por más de 70 años como antagonista para el control de enfermedades en plantas producidas por hongos en el mundo (Kubicek \& Harman 2002).

Entre los MS de Trichoderma spp. se encuentra el ácido-3-indolacético (AIA) una hormona inductora del crecimiento (Gravel et al. 2007), así Trichoder ma spp. como hongo promotor del crecimiento (PGPF: Plant Growth Promoting Fungi) (Yedidia 1999, Harman 2000, Gravel et al. 2007, Vinale et al. 2008, Achá 2008), acción que fue estudiada mediante la inducción de la producción de metabolitos con (i) aminoácidos inductores específicos de hormonas (AIA) como el L-triptófano (L-Trp) (Gravel et al. 2007), (ii) patógenos (Vinale et al. 2006, Vinale et 17 al. 2008) y (iii) el inóculo directo de Trichoderma spp. identificado como PGP. Se ha logrado incrementar la velocidad y porcentaje de germinación, reducir los efectos causados por condiciones de estrés ambiental (Bjorkman et al. 1998), y frente a patógenos como Rhizoctonia solani y Fusarium sp. comunes en cultivos agrícolas (Achá 2008), como también el aumento de la altura de las plantas, peso seco y tamaño de las raíces (Kleifeld \& Chet 1992). Así, la creciente demanda de alternativas ecológicas para el uso de agroquímicos y fertilizantes sintéticos en la producción agrícola, su estudio de propiedades benéficas de los microorganismos en el suelo (Har man et al. 2004, Vinale et al. 2008, Vinale et al. 2009, Ryder et al. 2012). Actualmente se desarrollan productos con organismos vivos, conocidos como agentes de control biológico: hongos, bacterias, virus e insectos (PROINPA 2012) a través del aislamiento y caracterización de microorganismos nativos bolivianos mediante pruebas en laboratorio (Ortuño et al. 2004) y en campo mediante ensayos realizados con Trichoderma spp. en plántulas de cebolla y tomate en el Valle Alto de CochabambaBolivia donde se destaca la particularidad de este hongo co mo PGPF (Medrano \& Ortuño 2007, Achá 2008).

Por esta razón, las cepas de Trichoderma spp. han adquirido un alto valor comercial, abarcando nuevas tecnologías para la producción masiva de productos de este hongo (Cruz 2007). Aunque su uso través de productos donde se tiene como componente activo esporas de Trichoderma spp. en diferentes medios de crecimiento, ya sea de forma sólida o líquida (PROINPA 2012), se tiene como dependencia la capacidad de crecimiento y supervivencia del hongo 
en el sustrato inoculado y la competencia con otros micro organismos de suelo.

Esta interacción provocada lleva a una mayor producción de componentes de defensa, sintetizados a través del metabolismo secundario del hongo donde se ha encontrado al AIA entre otros (Benítez et al. 2004). Según Kilian et al. (2006), el desarrollo de productos biológicos basados en microorganismos benéficos puede ampliar las opciones del mantenimiento y rendimiento de cultivos en campo, dentro de la agricultura boliviana andina, la baja fertilidad, deficiente conservación de suelos se refleja en bajos rendimientos de los cultivos, que sería superable con el empleo de biofertilizantes de preparación local (Franco et al. 2005).

Por consiguiente se ha visto necesario la búsqueda de formas alternativas de obtener dichos beneficios: mediante técnicas de estrés biológico en laboratorio, como los enfrentamientos duales de Trichoderma spp. donde se aprovecha doblemente el potencial del hongo, identificándose las cepas productoras de MSPGP dejando de lado los métodos tradicionales de enfrentamientos de Trichoderma spp. con patógenos de la planta.

\section{Materiales y métodos}

El ensayo se realizó en el laboratorio de microbiología de la Fundación para la Promoción e Investigación de Productos Andinos (Fundación PRO INPA), ubicada en la provincia Quillacollo del departamento de Cochabamba, que se encuentra a $15 \mathrm{Km}$ de la ciudad, a una altitud de 2613 msnm y cuyas coordenadas geográficas son $17^{\circ} 21^{\prime} 00.7^{\prime \prime}$ latitud sur y 66 15'40.6" longitud oeste.

Cepas microbianas, 35 cepas nativas bolivianas de Trichoderma spp disponibles en la colección de la Fundación PROINPA, fueron activadas en (PDA: potato-dextrosa-agar, Difco ${ }^{\mathrm{TM}}$ Laboratories, Becton Dickinson, Sparks, MD, USA). Estas fueron incuba das en tubos de ensayo de $6 \mathrm{~mL}$ a $20 \pm 2{ }^{\circ} \mathrm{C}$ durante 8 días.

Prueba preliminar de detección del ácido indol acético (AIA), la capacidad de síntesis del ácido indol acético (AIA) de las 35 cepas de Trichoderma spp. de forma individual, Rico (2009). Inicialmente, se preparó el medio de cultivo caldo de papa (PDB: potato-dextrose-broth), según el protocolo establecido por el laboratorio de microbiología de la Fundación PROINPA. El medio fue llevado a la autoclave durante $15 \min$ a $121{ }^{\circ} \mathrm{C}$, se agregó la mezcla previamente filtrada de $0.153 \mathrm{~g}$ de L-Trp disuelto en 15 $\mathrm{mL}$ de agua. A continuación se realizó la inoculación de las 35 cepas de Trichoderma spp en erlenmeyer de $50 \mathrm{~mL}$ con el medio de cultivo PDB enriquecido con L-Trp (aminoácido precursor para la síntesis del AIA durante el desarrollo de Trichoderma spp.). El medio de crecimiento del hongo se mantuvo en agitación constante a $100 \mathrm{rpm}$ a $23 \pm 2{ }^{\circ} \mathrm{C}$ durante 7 días, se centrifugó a $5000 \mathrm{rpm}$ durante 10 min, en una centrífuga marca Vortex $21 \mathrm{~K}$, el sobrenadante se dispenso en placas de plástico tipo ELISA, con 96 hoyos de $250 \mu \mathrm{L}$, marca MicroWell. Para su revelado se adicionó solución de Salkowski $\left(\mathrm{ClFe}_{3}=2 \%\right.$ al $\left.0.05 \mathrm{M}, \mathrm{H}_{2} \mathrm{SO}_{4}=35 \% \mathrm{H}_{2} \mathrm{O}=63 \%\right)$, que detecta derivados del Indol (Gordon \& Weber 1951). Como testigo negativo o cero se usó un pocillo control con PDB más la solución de Salkowski. Para el control positivo se usaron dos bacterias identificadas (Bacillus subtilis y Bacillus amiloliquefasciens) como positivas a la prueba. La placa de ELISA se llevó a oscuridad por $15 \mathrm{~min}$, para su posterior revelado, se observó viraje de color de amarillo a diversas tonalidades entre el rango de rosado a fucsia encendido, coloración que indica la presencia de AIA (Gordon \& Weber 1951). 
Enfrentamientos duales-PDA, para seleccionar las mejores cepas de Trichoderma spp. productoras de MS de interés, se produjo el estrés biológico del hongo mediante enfrentamientos duales en placas petri de $90 \mathrm{~mm}$ de diámetro con PDA, las 35 cepas de Trichoderma spp. tuvieron en 7 días de crecimiento (placa matriz), de esta se tomó una muestra general de inóculo un disco de $5 \mathrm{~mm}$ de diámetro (PDA más hongo en crecimiento), que fueron sembrados en puntos equidistantes en placa petri de 50 $\mathrm{mm}$, se incubo a $20 \pm 2{ }^{\circ} \mathrm{C}$ por 8 días. Durante este tiempo, se realizaron controles de velocidad de crecimiento, compatibilidad o incompatibilidad. Hecho que se verifica con la presencia o no de la "barrera de defensa". Para este ensayo se trabajó con 315 enfrentamientos: combinaciones duales de las 35 cepas de Trichoderma spp. cada enfrentamiento contó con tres repeticiones.

Análisis estadístico, se consideraron los siguientes parámetros como variables de respuesta: Para el con trol de velocidad de crecimiento de los enfrentamientos duales, se tomó como variables (i) crecimiento horizontal del hongo en la placa petri $(\mathrm{cm}) \mathrm{y}$ (ii) crecimiento vertical del hongo en la placa petri $(\mathrm{cm})$, datos que fueron evaluados mediante un análisis de cluster con el programa estadístico PAST. Se observaron tres características de forma general al final de los 8 días de crecimiento: (i) compatibilidad de ciertas cepas durante su crecimiento en la placa petri, (ii) crecimiento en la placa petri de forma proporcional, y (iii) crecimiento agresivo de una de las dos cepas enfrentadas, limitando el avance de su antagonista en la placa petri. Al terminar el periodo de crecimiento del hongo, se tomó como parámetro de selección el ancho de barrera presentado en la placa petri (Tabla 1).
Tabla 1 Características de selección en los enfrentamientos de Trichoderma spp.

\begin{tabular}{ccc}
\hline $\begin{array}{c}\text { Ancho de } \\
\text { barrera }(\mathrm{cm})\end{array}$ & Selección & Características \\
\hline \hline 0.7 & Positiva* & Incompatibilidad. Barrera gruesa. \\
$0.5-0.6$ & Positiva* & Incompatibilidad. Barrera gruesa. \\
$0.4-0.5$ & Negativa & Incompatibilidad. Barrera delgada. \\
$0.2-0.3$ & Negativa & Incompatibilidad. Barrera delgada. \\
$0.1-0.2$ & Negativa & Incompatibilidad. Barrera fina. \\
0 & Negativa & Compatibles. No presenta barrera.
\end{tabular}

*Selección Positiva: indica que el enfrentamiento dual será replicado para la producción de MS en medio de cultivo PDB.

Producción de Metabolitos Secundarios (MS), la replicación en medio de cultivo PDB los 20 enfrentamientos duales que presentaron formación de la "barrera de defensa gruesa" $(0.5-0.7 \mathrm{~cm})$ en el medio de cultivo PDA y así facilitar la separación de la sustancia de interés producida durante la interacción de ambas cepas del micelio formado, realizándose nuevos cultivos duales con los enfrentamientos seleccionados según la metodología Rea ves \& Crawford (1994), también descrita en Vinale et al. (2009), en matraces erlenmeyer de $50 \mathrm{~mL}$ con PDB autoclavado a $121{ }^{\circ} \mathrm{C}$ durante 15 min, también se realizó el cultivo individual (discos de $5 \mathrm{~mm}$ de diámetro tomados de la plaza matriz) en PDB de las 12 cepas de Trichoderma spp. que forman parte de los enfrentamientos duales. Los matraces fueron llevados a agitación constante, $100 \mathrm{rpm}$ durante 9 días a temperatura ambiente $\left(25 \pm 2{ }^{\circ} \mathrm{C}\right)$. Cada enfrentamiento contó con cinco repeticiones, de ellas se tomó un matraz al azar para la medición del $\mathrm{pH}_{\text {inicial }}$ (día uno), $\mathrm{pH}_{\text {intermedio }}$ (a cuatro días), $\mathrm{pH}_{\text {final }}$ (a nueve días. Los cultivos individuales, con una repetición, se 
tomó el pH final de cada uno (datos no presentados).

Filtrado del medio de cultivo PDB, después de 9 días de crecimiento, los enfrentamientos y cultivos individuales de Trichoderma spp. en PDB, se separaron de la sustancia de interés del micelio del hongo formado mediante el siguiente protocolo: (i) se separó el micelio formado por Trichoderma spp. del caldo, (ii) se realizó choque térmico a $50{ }^{\circ} \mathrm{C}$ por 15 min del líquido obtenido, luego se filtró y separo cualquier resto de estructura fúngica de la sustancia de interés que contiene a los MS: (i) papel filtro $\mathrm{N}^{\circ} 1$, (ii) papel de filtro cualitativo $415 \mathrm{VWR}$ equivalente a papel filtro WHATMAN $\mathrm{N}^{\circ} 6$ de $3 \mu \mathrm{m}$ y (iii) Sterile Syringe Filter de $0.2 \mu \mathrm{m}$ Cellulose Acetato VWR mas una jeringa estéril de $5 \mathrm{~mL}$.

Bioensayos en invernadero, se utilizó el diseño alea torio por bloques, con 6 bloques para cada ensayo (Lactuca sativa y Raphanus sativus) cada bloque con 35 tratamientos: 20 enfrentamientos duales, 12 cepas individuales, 1 testigo $(\mathrm{T})$ caldo de cultivo PDB, 1 tratamiento biológico (BIO): TRICOBAL, y 1 tratamiento químico (QUI): INKAFERT AZUL. Los fertilizantes químico (QUI) y biológico (BIO) fueron instalados como tratamientos positivos, las semillas de Lactuca sativa y Raphanus sativus sem bradas en macetas de $2 \mathrm{~kg}$ con tierra estéril (ArenaCascarilla de Arroz-Tierra vegetal en proporción 1:1:1), esterilizada a $150{ }^{\circ} \mathrm{C}$ por 120 min con vapor de agua. A partir de la germinación de las se millas se controló el tiempo en el cual estas llegaron al estadio de plantines (punto de partida del ensayo). De esta forma se procedió a inocular $500 \mu \mathrm{L}$ de cal do filtrado de los enfrentamientos duales de Trichoderma spp. $500 \mu \mathrm{L}$ de caldo filtrado de las cepas individuales de Trichoderma spp. $0.5 \mathrm{~g}$ de TRICOBAL y $0.44 \mathrm{~g}$ de INKAFERT AZUL. El inóculo del caldo filtrado de $500 \mu \mathrm{m}$ por tratamiento se hizo en la zona de la meso-rizósfera. En el caso del inóculo sólido como es TRICOBAL e INKAFERT AZUL se realizó alrededor de la rizósfera entre 6-8 $\mathrm{cm}$ de profundidad.

Variables de respuesta, se realizaron evaluaciones semanales de desarrollo de plantines en cuanto a altura del área foliar, número de hojas, el regado de las plantas en desarrollo se realizó cada 48 h, por la mañana. Al cabo de un mes y medio de crecimiento controlado, se realizó la cosecha de Lactuca sativa y Raphanus sativus, se consideró las variables de respuesta: (i) cultivo de Lactuca sativa: peso de hoja (g), peso de raíz (g), largo de raíz (cm), volumen de raíz (mL) y (ii) cultivo de Raphanus sativus: peso de hoja $(\mathrm{g})$, diámetro del bulbo $(\mathrm{cm})$, peso del bulbo $(\mathrm{g})$, largo del bulbo $(\mathrm{cm})$, volumen del bulbo $(\mathrm{mL})$, largo de raíz $(\mathrm{cm})$, peso de raíz $(\mathrm{g})$.

Análisis estadístico, el análisis de cluster mediante el programa estadístico PAST para la evaluaciones de invernadero: cosecha de los cultivos de Lactuca sativa y Raphanus sativus. También se realizaron para los datos de las variables de respuesta obtenidos de la cosecha de los ensayos evaluaciones mediante el análisis de varianza (ANOVA) bajo la distribución normal con un nivel de confianza del 99\% con el software estadístico SAS-9.2.

\section{Resultados}

Prueba preliminar de detección de AIA, las 35 cepas de Trichoderma spp. analizadas de forma individual en la prueba de AIA, las cepas 1, 6, 7, 8, 10, $15,18,23,24,25,28,29,31,32$ marcaron positivo a la presencia de AIA.

Enfrentamientos duales-PDA, los controles de crecimiento de cepas Trichoderma spp. se observó un efecto antagónico en PDA, la formación de barrera de defensa entre cepas incompatibles en área de 
terminada de crecimiento. En su inicio una cepa enfrentada es capaz de inhibir a su antagonista a distancia, luego la detención, se realiza por secreción de sustancias antifúngicas, parámetro de selección, formación y dimensión de la barrera (Figura 1). Considerando la clasificación de los 105 enfrentamientos, realizados en PDA, se tiene como resultado: 20 enfrentamientos con barrera gruesa (Tabla 2) seleccionados para la producción de MS en PDB, además de ser este el grupo que mostró la menor velocidad de crecimiento al momento de competir frente a otro hongo del mismo género en un área determinada, según el análisis de Cluster.

Dentro de los 20 enfrentamientos duales de Trichoderma spp. las cepas individuales $1,6,7,15,18,23$, $24,25,28,29,31,32$ identificadas como productoras de AIA mediante la inducción del L-Trp, formaron la barrera de defensa gruesa al ser enfrentadas entre las cepas del mismo grupo en PDA, las cepas individuales 8 y 10 no presentaron el mismo resultado, ya que al ser enfrentadas con otra cepa de Trichoderma spp. del mismo grupo, la barrera de defensa formada en la placa petri con el medio de cultivo PDA fue delgada en un 56\% de los enfrentamientos y fina en un $36 \%$.

Producción de $M S$, la evaluación del pH final de los enfrentamientos duales y las cepas individuales de Trichoderma spp. en PDB, el análisis ANOVA indica que no existen diferencias estadísticas notables $(\mathrm{P}<0.01)$ entre ambos tratamientos. En general, el $\mathrm{pH}$ final de los cultivos de cepas individuales de Trichoderma spp. al final del ensayo presentó variaciones entre 4.28 y 8.38 , y solamente una cepa presentó pH neutro. Asimismo el pH final de los enfrentamientos duales no sobrepasa el valor de 7.17.
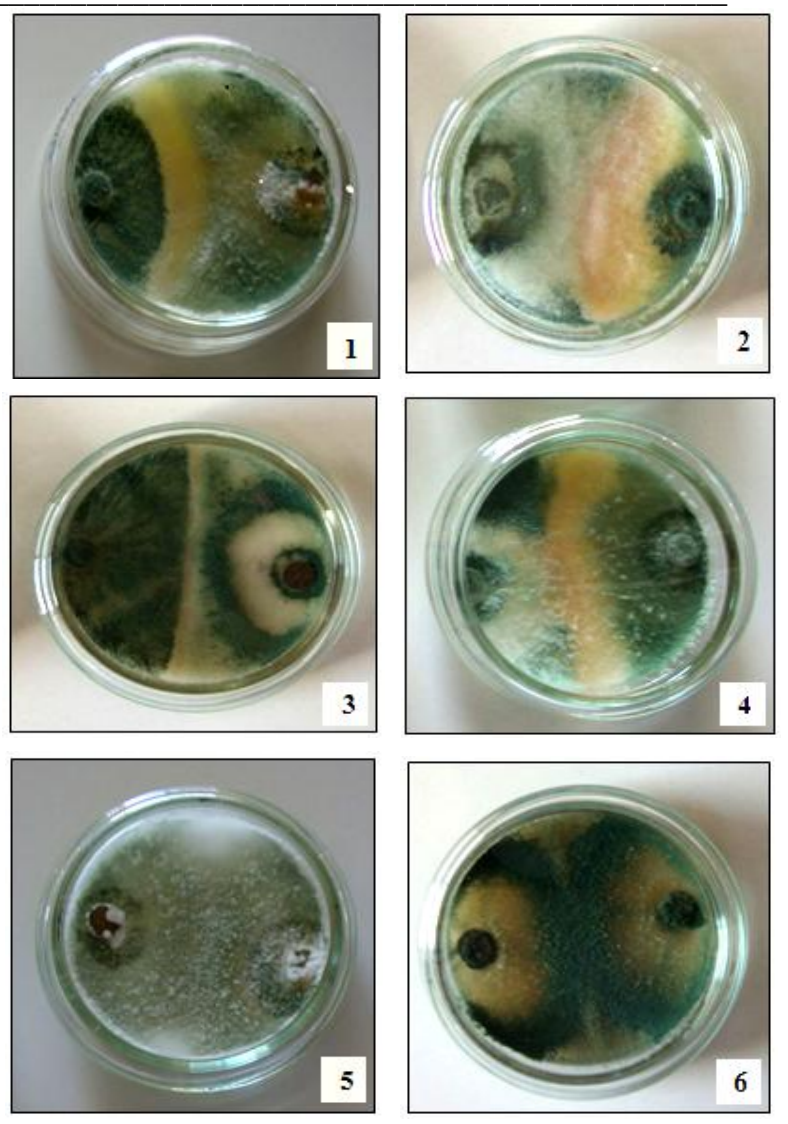

Figura 1 Clasificación de enfrentamientos duales. 1: Menor desarrollo de la cepa A frente a la cepa B, 2: La cepa A se desarrollo en mayor area que la cepa B con ataque al hongo antagonista, 3: Crecimiento compartido de las dos cepas, con formación de barrera, 4: Ambas cepas se desarrollaron de igual forma pero la cepa $\mathrm{A}$ ataca fuertemente a la cepa B, 5: Cepas compatibles sin tinción del medio de cultivo PDA, 6: Compatibles con tinción de medio de cultivo PDA.

\section{Bioensayos en invernadero}

Análisis estadístico para el ensayo de Lactuca sati$v a$, se muestra que los enfrentamientos duales y los cultivos individuales, pueden ser agrupados en 8 (Tabla 3) según la eficiencia de acción como PGPF. Por lo tanto, de acuerdo al análisis de las medias generales para las variables de respuesta evaluadas al final de la cosecha, los mejores tratamientos pertenecen al "Grupo 5" con los tratamientos: 3, 4, 7 (enfrentamientos) e I (cultivo individual). Por lo que 
estos tratamientos podrían utilizarse como promoto-

res de crecimiento en el cultivo de Lactuca sativa

(Figura 2).

Tabla 2 Clasificación de los enfrentamientos duales de Trichoderma spp. según la velocidad de crecimiento: análisis de Cluster

\begin{tabular}{ccll}
\hline $\begin{array}{c}\text { Grupo según } \\
\text { Cluster }\end{array}$ & Velocidad de crecimiento & \multicolumn{2}{c}{ Enfrentamientos duales* } \\
\hline Grupo 1 & Mayor velocidad de crecimiento & Compatibles: & 47 enfrentamientos \\
Grupo 2 & Grupo 2 < Grupo 1 & Barrera fina: & 27 enfrentamientos \\
Grupo 3 & Grupo 3 < Grupo 2 & Barrera delgada: 11 enfrentamientos \\
Grupo 4 & Menor velocidad de crecimiento & Barrera gruesa: 20 enfrentamientos \\
\hline *Clasificación de los enfrentamientos duales de Trichoderma spp. según la presencia de barrera.
\end{tabular}
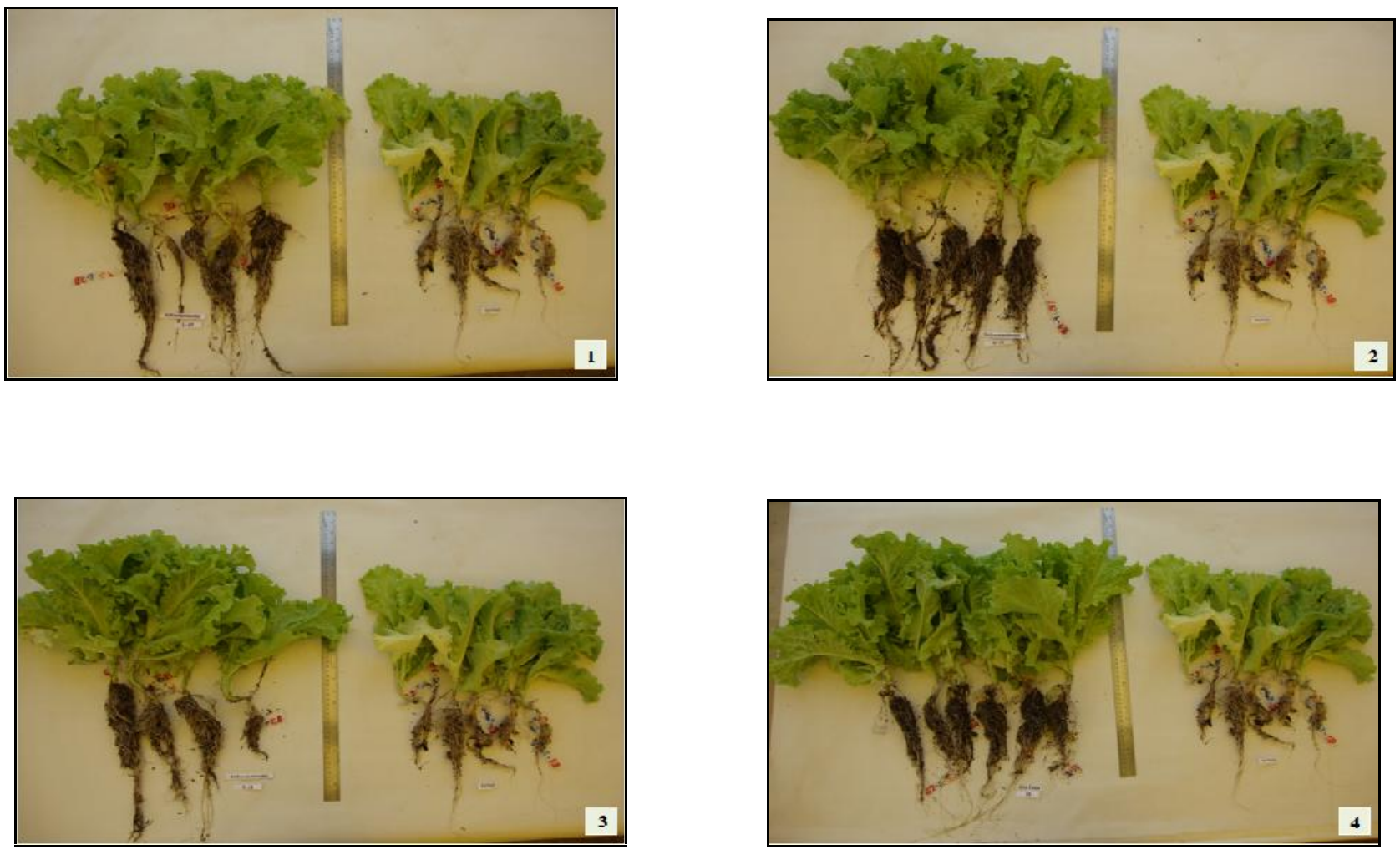

Figura 2 Lactuca sativa-PGPF "Grupo 5". 1: Enfrentamiento 3 comparado con el Testigo T, 2: Enfrentamiento 4 comparado con el Testigo T, 3: Enfrentamiento 7 comparado con el Testigo T, 4: Cultivo individual I comparado con el Testigo T. 
Tabla 3 Medias generales para las variables de crecimiento y rendimiento de Lactuca sativa según el análisis de Cluster

\begin{tabular}{cccccc}
\hline $\begin{array}{c}\text { Grupo según } \\
\text { Cluster }\end{array}$ & Tratamientos & $\begin{array}{c}\text { Peso de } \\
\text { hoja }(\mathrm{g})\end{array}$ & $\begin{array}{c}\text { Peso de } \\
\text { raíz }(\mathrm{g})\end{array}$ & $\begin{array}{c}\text { Largo de } \\
\text { raíz }(\mathbf{c m})\end{array}$ & $\begin{array}{c}\text { Volumen de } \\
\text { raíz }(\mathbf{m L})\end{array}$ \\
\hline $\mathbf{1}$ & 12-A-B-13-C & 1.696 & 0.673 & 7.129 & 1.006 \\
$\mathbf{2}$ & 20 & 7.315 & 1.568 & 16.656 & 1.566 \\
$\mathbf{3}$ & 6-17-9-18-10-8-2-QUI & 63.730 & 3.026 & 27.750 & 3.069 \\
$\mathbf{4}$ & 16-K-1-19-F-H-BIO-J & 52.359 & 2.887 & 29.004 & 3.132 \\
$\mathbf{5}^{*}$ & $4-7-3-\mathrm{I}$ & 67.582 & 3.802 & 39.979 & 3.813 \\
$\mathbf{6}$ & 5 & 42.134 & 2.795 & 20.659 & 2.555 \\
$\mathbf{7}$ & 11-14-L-T-15-G & 32.511 & 2.524 & 27.379 & 2.556 \\
$\mathbf{8}$ & $\mathrm{E}$ & 20.280 & 1.177 & 15.069 & 1.304 \\
\hline
\end{tabular}

*Grupo 5: Mejores PGPF.

Por otra parte en análisis de varianza muestra que se encontraron diferencias estadísticas significa tivas $(\operatorname{Pr}<0.01)$ para las variables de respuesta peso de hoja, peso de raíz, largo de raíz y volumen

\section{Análisis estadístico para el ensayo de Raphanus} sativus, se muestra que los enfrentamientos duales y los cultivos individuales, pueden ser agrupados en 9 (Tabla 4). De los cuales el mejor grupo como PGPF, de acuerdo al análisis de medias generales para las variables de respuesta evaluadas al final de la cosecha, es el "Grupo 6" (Figura 3) con los tratamientos $\mathbf{1 0}$ (enfrentamiento) y $\mathbf{K}$ (cultivo individual).
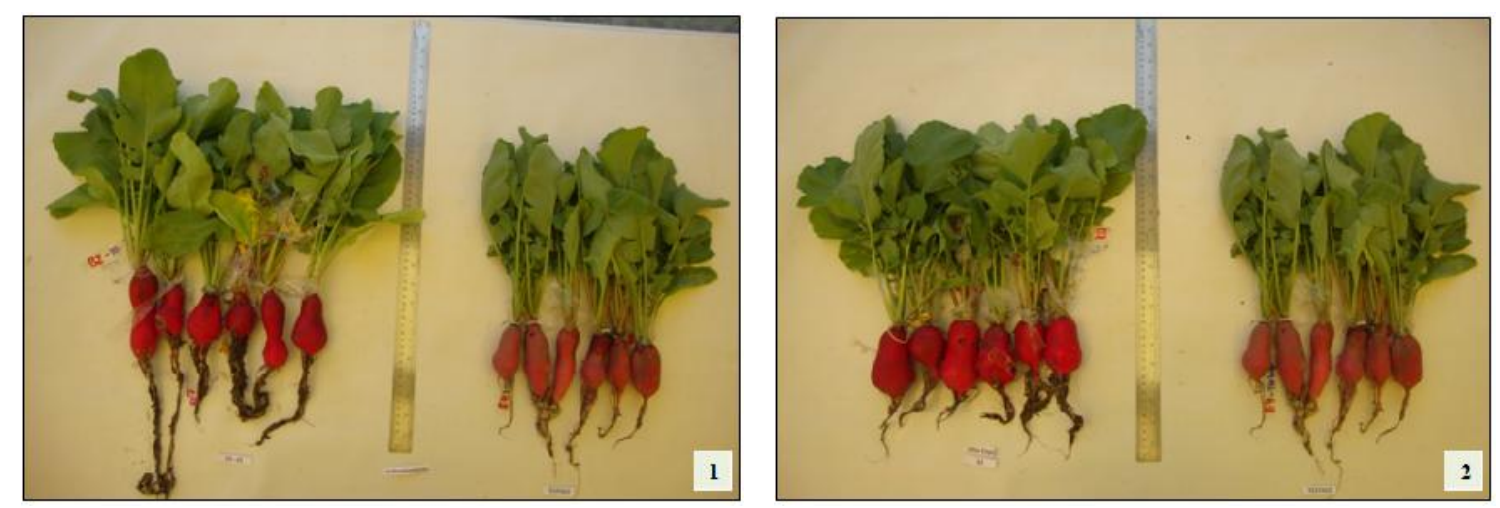

Figura 3 Raphanus sativus-PGPF "Grupo 6". 1: Enfrentamiento 10 comparado con el Testigo T; 2: Cultivo individual K comparado con el Testigo $\mathrm{T}$. de raíz para el cultivo de Lactuca sativa. Por lo que el efecto de los cultivos individuales y enfrentamientos duales entre los tratamientos varió según los caracteres de la planta.

El análisis de varianza muestra diferencias signi ficativas $(\operatorname{Pr}<0.01)$ para las variables de respuesta peso de hoja, diámetro de bulbo, peso de bulbo, largo de bulbo, volumen de bulbo y largo de raíz para el cultivo de Raphanus sativus, y no así para la variable peso de raíz. 
Tabla 4 Medias generales para las variables de crecimiento y rendimiento de Raphanus sativus según el análisis de Cluster

\begin{tabular}{|c|c|c|c|c|c|c|}
\hline Grupo según Cluster & Tratamientos & $\begin{array}{l}\text { Peso de } \\
\text { hoja (g) }\end{array}$ & $\begin{array}{l}\text { Diámetro de } \\
\text { bulbo }(\mathbf{c m})\end{array}$ & $\begin{array}{c}\text { Peso de } \\
\text { bulbo (g) }\end{array}$ & $\begin{array}{l}\text { Largo de } \\
\text { raíz }(\mathrm{cm})\end{array}$ & $\begin{array}{l}\text { Peso de raíz } \\
\text { (g) }\end{array}$ \\
\hline 1 & A & 4.862 & 3.183 & 28.242 & 28.333 & 0.723 \\
\hline 2 & G-QUI & 5.656 & 3.175 & 35.648 & 30.833 & 0.737 \\
\hline 3 & $\mathrm{~L}$ & 3.566 & 2.197 & 14.040 & 15.833 & 0.695 \\
\hline 4 & $5-8-6-\mathrm{H}-11-7-2-16$ & 5.380 & 3.992 & 58.360 & 58.271 & 0.981 \\
\hline 5 & 9-BIO-E & 5.277 & 3.806 & 52.879 & 52.778 & 0.884 \\
\hline 6 & $10-\mathrm{K}$ & 5.555 & 4.142 & 66.447 & 69.583 & 1.344 \\
\hline 7 & 4-12-I-13-F-T-17-19-14-15-18-B-D-1 & 5.229 & 3.575 & 46.237 & 46.012 & 1.063 \\
\hline 8 & 20-J-C & 4.923 & 3.372 & 41.518 & 38.889 & 0.916 \\
\hline 9 & 3 & 5.508 & 4.083 & 53.593 & 41.250 & 1.657 \\
\hline
\end{tabular}

*Grupo 6: Mejores PGPF.

\section{Discusión}

Prueba preliminar de detección de AIA, en la prueba de detección de AIA catorce cepas individuales de Trichoderma spp. presentaron el viraje de color rojo-rosado, indicando la presencia de AIA. Según lo reportado por Salkowski 1889, Bric et al. 1991, Glickmann \& Dessaux 1995, Anwar 2000, su detección en el medio permite establecer si el microorganismo de interés tiene la capacidad de sintetizar AIA (hormona de crecimiento vegetal) a partir de un precursor determinado (L-Trp) y, así mismo, evaluar la especificidad encontrada en la reacción colorimétrica de Salkowski para la detección de compuestos indol. Es así que Yedidia et al. (1999), de mostró que monocultivos de Trichoderma spp. son responsables del incremento en el crecimiento de la planta de pepino (Cucumis sativus L.) en un ambiente aséptico. Gravel et al. (2007), también demostró que una cepa de Trichoderma; T. vatroviride es capaz de sintetizar AIA “in vitro" mediante la inducción de diferentes precursores como el L-Trp, triptamida y tryptofol
(200 $\mu \mathrm{g} \mathrm{mL}^{-1}$ ) en el medio de cultivo, mostrando que raíces de plántulas de tomate crecieron en presencia de concentraciones variantes de AIA (0$\left.10 \mu \mathrm{g} \mathrm{mL}^{-1}\right)$.

Producción de $M S$, de acuerdo a la selección, de los 20 enfrentamientos duales, a partir de la condición: mejores formadores de "barrera de defensa" zona en la que se presentó (i) inhibición de crecimiento por parte de una de las dos cepas inoculadas en la placa Petri y (ii) coloración de la zona de la barrera de defensa, Kubicek \& Harman (2002) indican que; algunos de los MS que pro duce este hongo presentan coloración en el medio de crecimiento, además de la característica pigmentación de ciertas cepas. Además, las cepas que pre sentaron mayor incompatibilidad con la formación de barrera de defensa mayor, presentan el desarrollo más lento durante los 8 días de crecimiento.

Por lo tanto, mientras los enfrentamientos duales presenten menor compatibilidad y mayor estrés durante su crecimiento más lento será su desarro- 
llo en un área determinada. Y ya que la producción de MS en hongos es un proceso complejo asociado al desarrollo morfológico (Vinale et al. 2009), y según Vessey (2003) la producción de reguladores del crecimiento de la planta por microorganismos es un mecanismo importante muchas veces asociado a la estimulación durante el crecimiento, se ha establecido como for ma de síntesis de los MS de interés el método de enfrentamientos duales de Trichoderma sp. Es así que en condiciones naturales como indica Sánchez et al. (2005), en el suelo, los microorganismos llegan a sintetizar mayor cantidad de AIA en comparación con cultivos puros en condiciones de laboratorio, posiblemente por efectos sinérgicos con otros microorganismos e interacciones con la materia orgánica del suelo. Por lo que el estrés causado a estas 12 cepas en enfrentamientos duales simula esta condición.

Ryder et al. (2012), descartan la posibilidad que el efecto PGP de Trichoderma spp. ocurra en respuesta al control de patógenos menores en la raíz, como se ha venido estudiando, ya que cepas de Trichoderma spp. si tienen efecto PGP en ausencia de alguna enfermedad detectable (Chang et al. 1986, Ryder et al. 2012) y en suelo o turba estériles (Windham et al. 1986, Ryder et al. 2012), condiciones en las que se instalaron los ensayos en invernadero para Lactuca sativa y Raphanus sativus. Por lo tanto, el efecto de PGP por el hongo no necesariamente ocurre a través de la liberación de nutrientes o control ejercido sobre alguna enfermedad causada por algún patógeno presente en la planta. Es así que se demuestra la capacidad que tienen cepas individuales de Trichoderma spp. de sintetizar MS-PGP, como el tratamiento I en Lactuca sativa y $\mathrm{K}$ en Raphanus sativus que fueron inoculadas en los ensayos en invernadero, en au25 sencia de estrés por enfermedad de la planta, patógeno o un competidor durante su crecimiento en el medio de cultivo PDB.

La cantidad de MS acumulados por Trichoderma spp. en los enfrentamientos duales con hongos patógenos depende del balance entre el efecto elicitador y la velocidad de biotransformación ejercida por un patógeno huésped (Vinale et al. 2009). Lo que indica que la cantidad de MS producida por Trichoderma spp. dependerá del estado del hospedero así como de los siguientes factores: (i) la cepa a considerar, (ii) el microbio elicitador o condición y (iii) el balance entre biosíntesis y biotransformación de los metabolitos (Vinale et al. 2009). Dada la interacción a la que fueron sometidas las cepas de Trichoderma spp. en los enfrentamientos duales, donde ellas actuaron como competidores de espacio, nutrientes y patógenos frente a otra cepa del mismo género, además de causar posible daño en estructuras, paredes celulares, frenar el crecimiento de la otra cepa antagonista como menciona Harman (2000). Asimismo en este estudio se evidenció que las cepas de Trichoderma spp. sintetizan MS con capacidad PGP de forma conjunta en enfrentamientos duales y de forma individual. Obteniendo mejores resultados con los inóculos de enfrentamientos duales en un $80 \%$ para el ensayo de Lactuca sativa y en un $82 \%$ para el ensayo de Raphanus sativus comparados con los inóculos de cepas individuales.

El entendimiento y manejo de los anteriores factores significaría una mejor comprensión del proceso de producción de estos compuestos naturales secretados por Trichoderma spp. durante los enfrentamientos. Y la aplicación directa, de estos compuestos o moléculas elicitadas, en cultivos vegetales representa un nuevo enfoque para el desarrollo de biofertilizantes basados en compues- 
tos bioactivos: MS como indica Vinale et al. (2009). Estos productos naturales: MS, han sido estudiados, identificados y aislados como muestra de la capacidad de Trichoderma spp. de biosintetizarlos y son aplicados como PGP en cultivos vegetales. Este hecho ha sido relacionado con el metabolismo secundario de este hongo a manera de favorecer la asociación simbiótica entre algunas cepas de Trichoderma-planta. (Kubicek \& Harman 2002, Harman et al. 2004, Vinale et al. 2008, Vinale et al. 2009, Lorito et al. 2010, Morán-Diez et al. 2012). Lo que respalda la hipótesis de tener AIA sintetizado en el caldo PDB empleado para los ensayos en invernadero debido al estrés biológico causado al enfrentar dos cepas de Trichoderma spp. por lo que este método es también una forma alternativa de producción de esta hormona.

Considerando lo reportado por Delgado-Jarana et al. (2000), el pH del ambiente de la rizósfera es uno de los factores que afectan la actividad de Trichoderma spp. y los factores patogénicos secretados por diferentes microorganismos. Por ejemplo algunos antibióticos son degradados a $\mathrm{pH}$ elevado, y en un $\mathrm{pH}$ bajo se podría inducir la degradación enzimática mediante proteasas ácidas. Aunque Trichoderma spp. tiene la habilidad de adaptarse a un amplio rango de condiciones extremas esta característica le da ventaja frente a otro organismo durante su interacción en un suelo ácido si se da el caso, incluso la adaptación de los compuestos bioactivos sintetizados cuentan con esta ventaja. Ya que los enfrentamientos y cultivos individuales presentan un amplio rango de crecimiento y por lo tanto la tolerancia de sus compuestos sintetizados es amplia.

Filtrado del caldo de cultivo PDB, Kamilova et al. (2006), indica que para realizar el aislado de los exudados de plántulas y semillas de tomate (Lyco- persicon esculentum L.) pepino (Cucumis sativus L.) pimienta (Capsicum annum L.), que contienen también MS, del medio de crecimiento "in vitro" estos fueron secados a $45^{\circ} \mathrm{C}$ y filtradas (tamaño de poro $0.45 \mu \mathrm{m}$ ) y el aminoácido Trp también fue extraído de la misma forma. Se realizó el choque térmico de $50{ }^{\circ} \mathrm{C}$ por 15 min lo que ayudó a desactivar las cepas de Trichoderma spp. Indicando que algunos de estos compuestos naturales: MS, resisten temperaturas altas por tiempo prolongado sin daño a sus estructuras, manteniendo su viabilidad. Además de considerar la efectividad del filtrado de la sustancias de interés que contiene a los MS. Ya que según Reyes et al. (2007), la obtención de filtrados puros del caldo de cultivo PDB de cepas de Trichoderma spp. mediante filtrados es una técnica útil de obtener dicha sustancia. Aunque también indica que pude haber ocurrido la destrucción o pérdida de algunos compuestos durante las fases previas a su extracción.

Bioensayos en invernadero, el AIA exógeno, como el producido por microorganismos, causa cambios en la morfología del sistema de la raíz el cual se ve influenciado en la toma de nutrientes para la planta (Arteca 1996). Ya que según indica Kamilova et al. (2006), la mayor parte de la auxina encontrada en la rizósfera de forma natural, es derivada del triptófano secretado por la raíz y convertida en auxina por bacterias y hongos. En cuanto a la inoculación del caldo filtrado de los enfrentamientos duales y cultivos individuales de Trichoderma spp. que fue aplicado a las plántulas jóvenes de lechuga (Lactuca sativa) y rábano ( $R a$ phanus sativus) se debe a que: el estado de plántula de una planta en desarrollo es más sensitiva a la estimulación de crecimiento vegetal por la auxina (Kamilova et al. 2006) u otra hormona de crecimiento o PGP. Pero la aplicación de la cantidad o 
concentración de estas sustancias: MS debe ser considerada seriamente ya que Contreras-Cornejo et al. (2009), indica que el efecto deseado mediante la inoculación de cepas de Trichoderma en la planta bajo condiciones naturales dependerá de la concentración y el tipo de auxinas que produzca el hongo, debido a que en pruebas realizadas con AIA y AIA1d inoculadas en plantas de Arabidopsis promovieron el crecimiento de la planta a concentraciones bajas. Caso que pudo pre sentarse en los tratamientos 7, 4, 3, 8 (enfrentamientos) e I (cultivo individual) en Lactuca sativa y $\mathbf{K}$ (cultivo individual) y 10, 8, 5, 11 (enfrentamientos) en Raphanus sativus. En cambio a concentraciones elevadas actuaron como represores del crecimiento (Acosta et al. 2001). Caso que pudo darse en el cultivo de lechuga inoculadas con los tratamientos 12, 13, 20 (enfrentamientos) y $\mathbf{A}, \mathbf{B}$ y $\mathbf{C}$ (cultivo individual) al presentar un porcentaje bajo en cuanto al crecimiento foliar y radicular, como también la muerte de las plantas inoculadas con el tratamiento D (cultivo individual). Siendo el cultivo de lechuga más sensible a los compuestos de interés: MS. Por lo tanto el AIA incluso el producido por microorganismos, puede influir en el crecimiento de sistemas radiculares dependiendo de la cantidad encontrada en la rizósfera a través de la elongación y formación de raíces laterales o adventicias (Taiz \& Zeiger 2006, Gravel et al. 2010). Y Trichoderma spp. produce auxinas que son capaces de estimular el crecimiento de la planta y desarrollo de la raíz (Contreras-Cornejo et al. 2009).

Ya que la inoculación de Trichoderma spp. de forma directa en la planta si tiene el efecto PGP de bido a las sustancias naturales sintetizadas por este hongo: MS, identificados por Kubicek \& Harman 2002, Vinale et al. 2008, como la harziano piridi27 na, 6PP, Koninginin, Koninginin A, Konin ginin B, Koninginin C, Koninginin E, Ciclo nerodiol y la presencia de auxinas (Contreras-Cornejo et al. 2009) que actúan como reguladores del crecimiento de la planta y además han sido encontrados dentro de los productos metabólicos de Trichoderma spp. Es así que se han realizado pruebas de forma directa y controlada, como Ryder et al. (2012) en lechuga, donde la actividad PGP se debe en parte a los compuestos solubles en agua, que fueron inoculadas con el líquido extraído del cultivo de la cepa Trichoderma hamatum GD12 capaces de incentivar el crecimiento de este vegetal. También Kamilova et al. (2006), demostró que en estudios previos realizados en invernadero, tratando a las semillas de una planta con una sus pensión de esporas de Trichoderma spp. incrementron el rendimiento de esta. Resultado similar se encontró al tratar las semillas separadas de Trichoderma spp. por una membrana de celofán, indicando que Trichoderma spp. produce metabolitos difusibles capaces de promover el crecimiento de la planta (Benítez et al. 1998). Indican también que algunos MS de Trichoderma spp. están directamente involucrados en la interacción planta-Trichoderma y particular mente que 6PP puede ser considerado como un compuesto similar a la auxina y/o puede actuar como un inductor de auxinas (Vinale et al. 2008).

La identificación de nuevos efectores moleculares pueden apoyar la aplicación de nuevos biofertilizantes basados en MS de Trichoderma spp. para ser utilizados en lugar de microorganismos estimuladores del crecimiento vegetal. Un punto de partida para lograr este cometido podría ser el estudio de la actividad de las cepas enfrentadas $\mathbf{3}$, 4, 5, 7, 8, 10, 11 y los cultivos individuales I, K que presentaron el mejor rendimiento de los 35 
tratamientos en los ensayos de invernadero. También Whipps (2001), menciona que ciertos aislados de Trichoderma spp. pueden actuar como PGPF en ausencia de patógenos mayores. Contreras-Cornejo et al. (2009) encontró que $T$. virens y $T$. atroviride son capaces de estimular el crecimiento en plantas "in vitro" de Arabidopsis. Debido al in cremento en el crecimiento de la raíz y profundidad de esta como consecuencia para una mejor absorción de nutrientes (Yedidia et al. 2001, Harman et al. 2004). Como se observó en los tratamientos 3, 4, 7, I, J en el cultivo de Lactuca sativa y los tratamientos 2, 10, 14, 19 en el cultivo de Raphanus sativus según el desarrollo en mayor proporción de la raíz.

El hecho de estimular a Trichoderma spp. enfrentándola con otra cepa del mismo género mediante la selección de los mejores productores o formadores de la barrera de defensa, asegura la producción de MS; hormonas que tengan la acción PGP, ya que según Whipps (2001), se debe considerar que si un microorganismo es capaz de crecer en la rizósfera, no proveerá de forma automática la acción PGP, ya que probado en el suelo no será siempre capas de colonizar la rizósfera o promover el crecimiento de la planta, esto por las variantes del suelo, factores bióticos y abióticos que influyen en la composición de las comunidades presentes en la zona de la rizósfera. Por ejemplo al detallar la interacción de las cepas enfrentadas de Trichoderma spp. con los códigos asignados a las cepas individuales se tiene a los mejores enfrentamientos que presentaron el mayor rendimiento en los ensayos de invernadero $\mathbf{3}(\mathbf{A}-\mathbf{J}), \mathbf{4}(\mathbf{B}-\mathbf{I})$, 5 (B-J), 7 (C-G), 8 (D-F), 10 (D-H), 11 (E-G). Dentro de estos enfrentamientos se cuenta con la participación de las cepas individuales $\mathbf{A}$, $\mathbf{B}, \mathbf{C}, \mathbf{D}, \mathbf{E}, \mathbf{F}, \mathbf{G}, \mathbf{H}, \mathbf{J}$ que no figuran entre las mejores inductoras del crecimiento de la planta. Pero se cuenta con una excepción en el enfrentamiento 4 donde se observa la presencia de la cepa individual I (una de las mejores cepas individuales inductora del crecimiento en el cultivo de Lactuca sativa). Por lo tanto el hecho de poder actuar como PGPF como cepa individual (cepas I, K) no asegura que estas se comportaran de la misma forma prometedora durante la interacción con otro hongo del mismo género. Igualmente cepas individuales con bajos rendimientos en cuanto a su acción PGPF, tienen el potencial para expresar mejor esta propiedad al ser inducidos por estrés biológico en un medio de crecimiento común (PDB), mejorando así las opciones de trabajo para elegir a las cepas sobresalientes como inductoras del crecimiento de la planta.

Es así que el ambiente juega un papel importante en la diferenciación y crecimiento de Trichoderma spp. La respuesta a estímulos exteriores durante el crecimiento, formación de conidias y microparasitismo, son tres características importantes que contribuyen al desarrollo de estos organismos, ya que el proceso de formación de conidias implica la detección del estímulo, comunicación intracelular, regulación de la expresión del gen de forma espacial y temporal y la especialización celular: cambios morfológicos (Carreras-Villaseñor et al. 2012). Dentro de los tratamientos utilizados en este estudio que rindieron mejor como PGPF son los enfrentamientos duales realizados, implicando una forma de estrés por competencia de nutrientes y espacio, entre dos cepas de Trichoderma spp. tanto en Lactuca sativa y Raphanus sativus y no los cultivos individuales (excepciones I, K).

Entre otras consideraciones, las observaciones realizadas al comportamiento de la fragilidad de ruptura y marchitamiento de las hojas, se advirtió 
que los cultivos de Lactuca sativa y Raphanus sativus tratados con el inóculo del caldo PDB que con tiene a los MS presentaron hojas más resistentes en comparación con el fertilizante químico INKAFERT-AZUL con un comportamiento de ruptura frágil. Además el marchitamiento se presentó más tempranamente con el fertilizante químico comparado con los tratamientos inocu ados con el caldo que contiene a los MS.

\section{Conflictos de interés}

Esta investigación y no presenta conflictos de interés.

\section{Agradecimientos}

Se agradece el apoyo económico del proyecto Fontagro-Bioinsumos y el Proyecto IP-Holanda.

\section{Literatura citada}

Achá C. Aislamiento y multiplicación de cepas nativas de Trichoderma sp y su evaluación como biocontrolador de Fusarium sp y Rhizoctonia solani en plantas de tomate. Tesis de Licenciatura en Ingeniería Ambiental. 2008; 1-94 pp.

Acosta M, Sánchez J, Bañón B. Auxinas. AzcónBieto J. \& Talón M. Fundamentos de fisiología vegetal. Editorial McGraw-Hill Interamericana, España. ISBN: 2001; 84-486-0258-7.261-283 pp.

Anwar G. Production of growth hormones and nitrogenase by diazotrophic bacteria and their effect on plant growth. PhD thesis, University of the Punjab, Lahore. 2000; 204 pp.
Arteca RN. Plant Growth Substances: Principles and Applications. Chapman \& Hall, New York. 1996; 332 pp.

Benítez T, Delgado-Jarana J, Rincón A, Rey M, Limón M. Biofungicides: Trichoderma as a biocontrol agent against phytopathogenic fungi. Citado en: Pandalai SG (eds) Recent research developments in microbiology. Research Signpost, Trivandrum. 1998; 2:129-150.

Benítez T, Rincón AM, Limon MC, Codón AC. Biocontrol mechanisms of Trichoderma strains. Department of Genetics, University of Sevilla, Spain. Int Microbiol. 2004; 7:249-260.

Bjorkman T, Blanchard LM, Harman GE. Growth enhancement of shrunfen-2 (sh2) Sweet Corn by Trichoderma harzianum 1295-22: Effect of Environmental Stress. J Amer Soc Hort Sci. 1998; 123(1):35-40.

Bric J, Bostock RM, Silverstone SE. Rapid In Situ Assay for Indol-acetic Acid Production by Bacteria Immobilized on a Nitrocellulose Membrane. Appl Environ Microbio. 1991; 535-538.

Carreras-Villaseñor N, Sánchez-Arreguin JA, Herrera-Estrella JA. Trichoderma: sensing the environment for survival and dispersal. Laboratorio Nacional de Genómica para la Biodiversidad. CINVESTAV Irapuato. Irapua to, Gto., México 2012. Microbiology. DOI: 10.1099/mic.0.052688-0. 158:3-16 pp.

Chang YC, Chang YC, Baker R, Kleifeld O, Chet I. Increased growth of plants in the presence of the biological control agent Trichoderma harzianum. Plant Dis. 1986; 70:145-148.

Contreras-Cornejo HA, Macías-Rodríguez L, Cortes-Penagos C, López-Bucio J. Trichoderma virens, a plant beneficial fungus, enhances biomass production and promotes lateral root growth through an auxin-dependent mechanism 
in Arabidopsis. Plant Physiol. 2009; 149:15791592.

Cruz L. Estandarización del proceso de producción masiva del hongo Trichoderma koningii Th003 mediante fermentación bifásica a escala piloto. Pontificia Universidad Javeriana. Bogotá, D.C. 2007; 133 pp.

Delgado-Jarana J, Pinto-Toro JA, Benitez T. Overproduction of beta-1,6-glucanase in Trichoderma harzianum is controlled by extracellular acidic proteases and $\mathrm{pH}$. Biochim Biophys Acta. 2000; 29:1481(2):289-96.

Druzhinina I, Kubicek CP. Species concepts and biodiversity in Trichoderma and Hypocrea: from aggregate species to species Clusters?. J Zhejiang. Univ Sci B. 2005;(2): 100-12.

Druzhinina IS, Seidl-Seiboth V, Herrera-Estrella A, Horwitz BA, Kenerley CM, Monte E, et al. Trichoderma: the genomics of opportunistic success. Nat Rev Microbiol. 2011; 9(10):749759.

Fernádez-Larrea O. Microorganismos para el control fitosanitario. Avance en el fomento de productos fitosanitarios no sintéticos. Costa Rica. 2001; 62:96-100.

Franco J, Herbas J, Ortuño N, Main G, Navia O. El Aporte de la Transformación de los Desechos Orgánicos en la Seguridad Alimentaria, Resultados de un Proyecto en Tiraque y Carrasco, Cochabamba. Revista de Agricultua. 2005; 34:14-18.

Glickmann E, Dessaux YA. Critical Examination of the Specificity of the Salkowski Reagent for Indolic Compounds Produced by Phytopathogenic Bacteria. Appl Environ Microbiol. 1995; 793-796.
Gordon SA, Weber RP. Colorimetric estimation of indoleacetic acid. Plant Physiol. 1951; 26(1):192-195.

Gravel V, Antoun H, Tweddell RJ. Growth stimulation and fruit yield improvement of greenhouse tomato plants by inoculation with $\mathrm{Pseu}$ domonas putida or Trichoderma atroviride: Possible role of indole acetic acid (IAA). Soil Biol Biochem. 2007; 39:1968-1977.

Harman GE. Myths and dogmas of biocontrol: changes in perceptions derived from research on Trichoderma harzianum T-22. Plant Disease. 2000; 84:377-393.

Harman GE, Howell CR, Viterbo A, Chet I, Lorito M. Trichoderma species-opportunistic, avirulent plant symbionts. Nat Rev Microbiol. 2004; 2:43-56.

Kamilova F, Kravchenko LV, Shaposhnikov AI, Azarova T, Makarova N, Lugtenberg B. Organic acids, sugars, and L-tryptophan in exudates of vegetables growing on stonewool and their effects on activities of rhizosphere bacteria. Mol Plant-Microbe Interact. 2006; 19:250256.

Kilian M, Steiner U, Krebs B, Junge $H$, Schmiedeknecht G, Hain R. FZB24® Bacillus subtilis-mode of action of a microbial agent enhancing plant vitality. Pflanzenschutznachrichten Bayer.;2006;1/00.1.

Kleifield O, Chet O. Trichoderma harzianuminteraction with living plants and effect on growth response. Plant Soil. 1992; 144:267272.

Kubicek C, Harman G. Trichoderma Gliocladium. Basic biology, taxonomy and genetics. Copyright Taylor \& Francis Ltd. 2002; 1:1-271.

Lorito M, Woo SL, Harman GE, Monte E. Translational research on Trichoderma: from omics 
to the field. Annu Rev Phytopathol. 2010; 48:395-418.

Medrano AM, Ortuño N. Control del Damping off mediante la aplicación de bioinsumos en almácigos de cebolla en el Valle Alto de Cochabamba-Bolivia Acta Nova. 2007; 3(4): 660679.

Morán-Diez E, Rubio B, Dominguez S, Hermosa R, Monte E, Nicolás C. Transcriptomic response of Arabidopsis thaliana after $24 \mathrm{~h}$ incubation with the biocontrol fungus Trichoderma harzianum. J Plant Physiolgy. 2012; DOI: 10.1016. 12:016.

Ortuño N, Navia O, Medrano A, Rojas K, Torrico L. Desarrollo de bioinsumos: Un aporte a la soberanía alimentaria de Bolivia. Revista de Agricultura. 2010; 62 (47):30-38.

Paleontological Statistics. Øyvind Hammer. Natural History Museum. University of Oslo.2011; Version 2.13.

PROINPA. Informes Anuales Promoción e Investigación de Productos Andinos. 2012; P.irr.

Reaves JL, Crawford RH. In vitro colony interactions among species of Trichoderma with inference toward biological control. United States Department of Agriculture, Forest Service (Pacific Northwest Research Station). 1994; 8 pp.

Reyes J, Pineda J, Sanabria ME. Efectividad de tres filtrados de hongos presentes en frutos de maíz (Zea mays L.) sobre Fusarium moniliforme. Agroecología. 2007; 2:47-53.

Rico M. Capacidad promotora de crecimiento vegetal por bacterias del género Azotobacter y Actinomicetos aislados de cultivos de Solanum tuberosum Linnaeus, 1753 (Papa) cultivados en zonas alto andinas del Perú. Facultad de Ciencias Biológicas. 2009; 138 pp.
Ryder LS, Harris BD, Soanes DM, Kershaw MJ, Talbot NJ, Thornton CR. Saprotrophic competitiveness and biocontrol fitness of a genetically modified strain of the plant-growth-promoting fungus Trichoderma hamatum GD12. Microbiol. 2012; 158(1):84-97.

SAS Institute Inc. The SAS System for Windows. Version 9.2. SAS Inst., Cary, NC. 2008; 141$179 \mathrm{pp}$.

Sánchez J, Valencia H, Valero N. Reducción de ácido indolácetico por microorganismos solubilizadores de fosfatos presentes en la rizósfera de Espeletia graniflora y Calamagrotis effuse del Páramo El Granizo. En: Bonilla M, Editor. Estrategias adaptativas de plantas del páramo y del bosque altoandino en la cordillera oriental de Colombia. Bogotá: Unibiblos. 2005; 177 $193 \mathrm{pp}$.

Salkowski E. Ueber die Bildung von Flüchtigen Fettsäuren bei der ammoniakalischen HarngÄhrung. Zeitschrift für Physiologische Chemie. 1989; 13:264-274.

Taiz L, Zeiger E. Plant Physiology. Cuarta Edición. Editorial Sinauer, New York. 2006; 690 pp.

Vessey JK. Plant growth promoting rhizobacteria as biofertilizers. Plant Soil. 2003; 255:571-586.

Vinale F, Marra R, Scala F, Ghisalbertic EL, Lorito M, Sivasithamparamb K. Major secondary metabolites produced by two commercial Trichoderma strains active against different phytopathogens. Lett Appl Microbiol. 2006; 43:143-148.

Vinale F, Sivasithamparamb K, Ghisalbertic E, Marraa R, Wooa S, Lorito M. Trichodermaplant-pathogen interactions. Soil Biol Biochem. 2008; 40:1-10. 
Vinale F, Sivasithamparamb K, Ghisalbertic E, Marra-Barbetti MJ, Li HR, Wooa S, et al. A novel role for Trichoderma secondary metabolites in the interactions with plants. Physiol Mol Plant Pathology. 2008; 72:80-86.

Vinale F, Ghisalbertic E, Sivasithamparamb K, Marra R, Ritieni A, et al. Factors affecting the production of Trichoderma harzianum secondary metabolites during the interaction with different plant pathogens. Lett Appl Microbiol. 2009; 48:705-711.

Vinale F, Flematti G, Sivasithamparamb K, Lorito M, Marra R, et al. Harzianic Acid, an Antifungal and Plant Growth Promoting Metabolite from Trichoderma Harzianum. J Nat Prod. 2009; 72:2032-2035.
Whipps JM. Microbial interactions and biocontrol in the rhizosphere. Journal of Experimental Botany, Roots Special Issue. 2001; 52:487-511.

Windham MT, Elad Y, Baker R. A mechanism for increased plant growth induced by Trichoderma spp. Phytopathology. 1986; 76:518-521.

Yedidia I, Benhamouand IN. Chet I. Induction of defense responses in cucumber plants $(\mathrm{Cu}$ cumis sativa L.) by the biocontrol agent Trichoderma harzianum. Appl Environ Microb. 1999; 65:1061-1070.

Yedidia I, Srivastva AK, Kapulnik Y, Chet I. Effect of Trichoderma harzianum on microelement concentration and increased growth of cucumber plants. Plant Soil. 2001; 235:235242. 